\title{
Method of signal processing in passive infrared detectors for security systems
}

\author{
H. Madura \\ Institute of Optoelectronics, Military University of Technology, \\ Warsaw, Poland
}

\begin{abstract}
This article presents the construction and principle of operation of passive IR detectors (PIR detectors) of a large detection range. An important virtue of these detectors is highly efficient detection of slowly moving or crawling people. The PIR detector described here detects crawling people at a distance of $140 \mathrm{~m}$. High signal-to-noise ratio was obtained by using a larger number of pyroelectric sensors, i.e., by using a larger number of detection zones (channels). The original electronic system for the PIR detector is presented in which DC signal amplifiers from pyroelectric signals are used. In order to ensure large detection ranges, a new method of signal analysis was used.
\end{abstract}

Keywords: PIR detector, security systems.

\section{Introduction}

Main elements of security systems are PIR detectors. In general, detectors operating inside buildings have small detection range, small ranges of working temperature, and relatively simple algorithms of intruder detection. The detectors used for protection of objects or large areas (buildings, airports) have larger detection ranges and complex algorithms of signal processing on which significantly depend the efficiency of their operation.

An essential drawback of currently available PIR detectors is low efficiency of detection of slowly moving or crawling people. It is because radiation from such objects is similar to background thermal noise. Moreover, to detect slowly moving or crawling people, the lower limit frequency of a transfer band of PIR detector should be near zero. By fulfilling this condition, increase in lowfrequency noise occurs causing a decrease in the next detector's sensitivity. 
Algorithms of intruder detection have to be different than these for typical PIR detectors. To detect crawling people, larger number of sensors should be used (more detection zones) which will cause an increase in signal-to-noise ratio because each of the sensors will "see" the smaller field of view.

PIR detectors used in security systems for people detection operate in far infrared spectral range $(8 \div 14 \mu \mathrm{m})$. In these detectors, the most frequently used are pyroelectric sensors allowing the detection of temperature changes as small as $10^{-6} \mathrm{~K}$. Application of a single pyroelectric sensor does not ensure distinguishing the events of an alarm nature from, so-called, false alarms caused by, e.g., air turbulences or background temperature changes resulting from sun radiation. Therefore in PIR detectors, the most frequently used are pyroelectric sensors with two active elements (two sensors) and an alarm signal is determined on the basis of analysis of a difference (or a sum) of their output signals $[1,2]$. Usually, pyroelectric sensors are mounted, together with a transistor and a resistor polarizing its gate, in standard hermetic housings. A value of this resistor can be even up to $10^{11} \Omega$, depending on the preamplifier configuration. JFET or MOSFET transistors are used most frequently as the amplifying elements that are mounted near a detector.

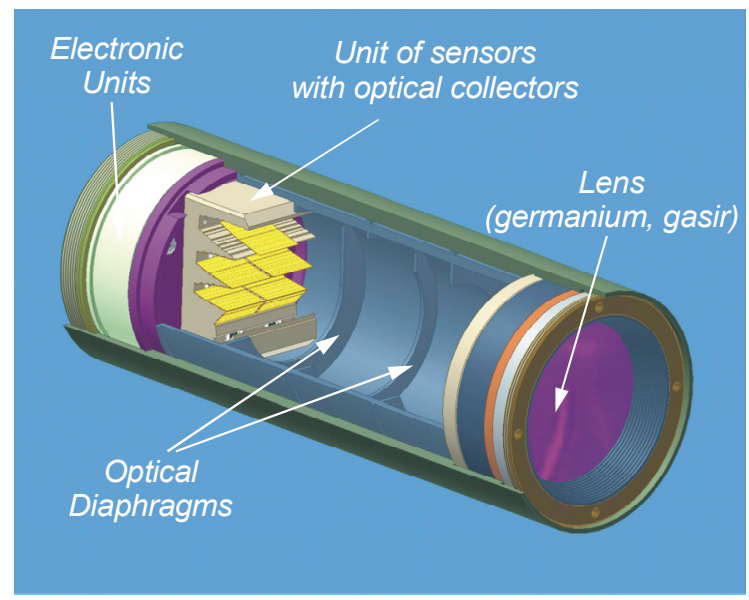

Figure 1: $\quad$ Simplified diagram of PIR detector.

\section{Construction of PIR detector}

Main elements of PIR detector are: objective (mirror or refraction one), set of pyroelectric sensors, and electronic systems (Fig. 1). The sensors convert an optical signal emitted from the "being observed" surface into an electrical signal. This signal is processed in the electronic systems (it is amplified, filtered, sampled) and next it is analysed in a microprocessor system.

The presented PIR detector detects the crawling people at a distance of 140 m. High signal-to-noise ratio was obtained due to application of the larger numbers of pyroelectric sensors, i.e., larger number of detection zones 
(channels). Application of larger number of sensors forces the necessity to develop a complex optical system (Fig. 2). The optical system of the PIR detector has to ensure such a position of the detection zones to the avoid presence of the areas which are "not seen" by a detector [3-5].
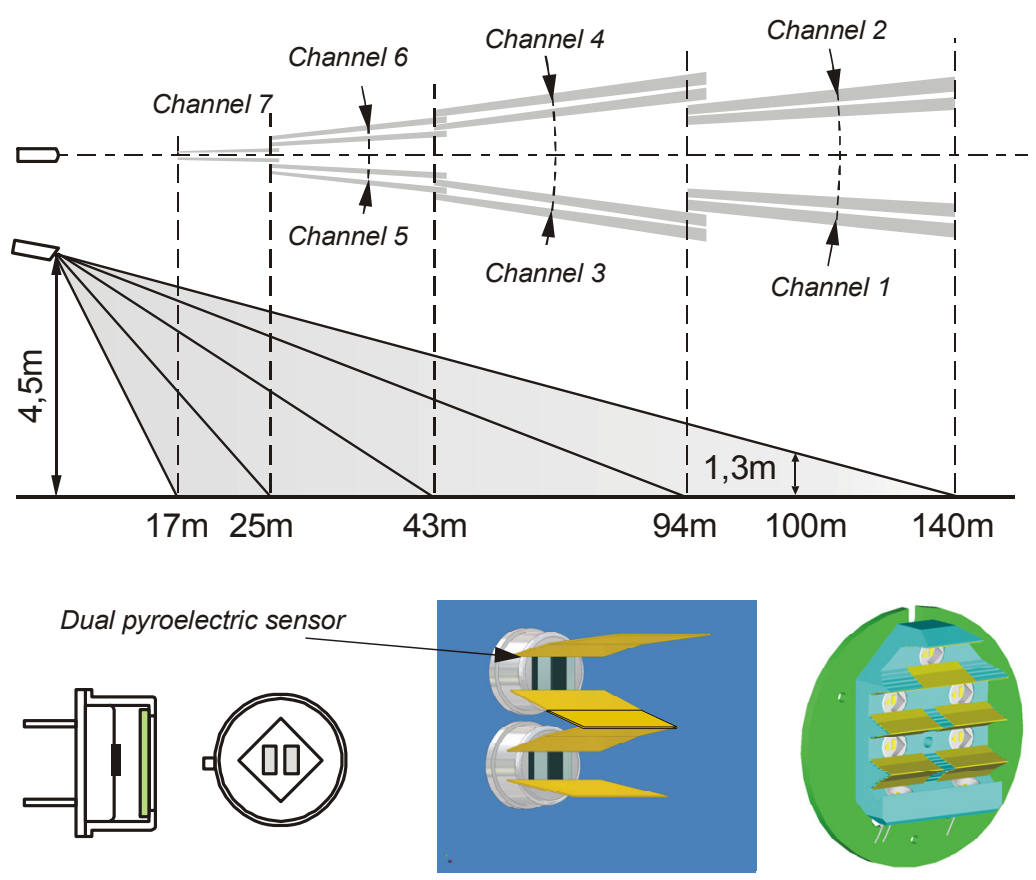

Figure 2: Detection zones of PIR detector in horizontal and vertical planes (top) and pyroelectric sensors with optical concentrators and general view of a detection set with seven two-segment sensors (bottom).

Radiation signals caused by slowly moving, especially crawling, people are characterized by similar luminance amplitudes and velocities of its change in time such as fluctuations of background radiation. The signal amplitude from pyroelectric sensors is directly proportional to the velocity of a change of radiation signal in time (i.e., to the velocity of a moving object). A disadvantageous property of pyroelectric sensors is a voltage drift (pyroelectric detector is equipped with a field transistor operating as a voltage follower) which at low velocities of intruder movement can have temporal characteristics of signals, comparable with the characteristics originating from a moving person. Thus, in order that the sensor could efficiently detect slowly moving person, it is necessary to develop an algorithm distinguishing both characteristic features of signal changes from a sensor caused by a photon noise and signal changes caused by a temperature drift of a pyroelectric sensor. A simplified diagram of the electronic circuit of PIR detector is shown in Fig. 3. 


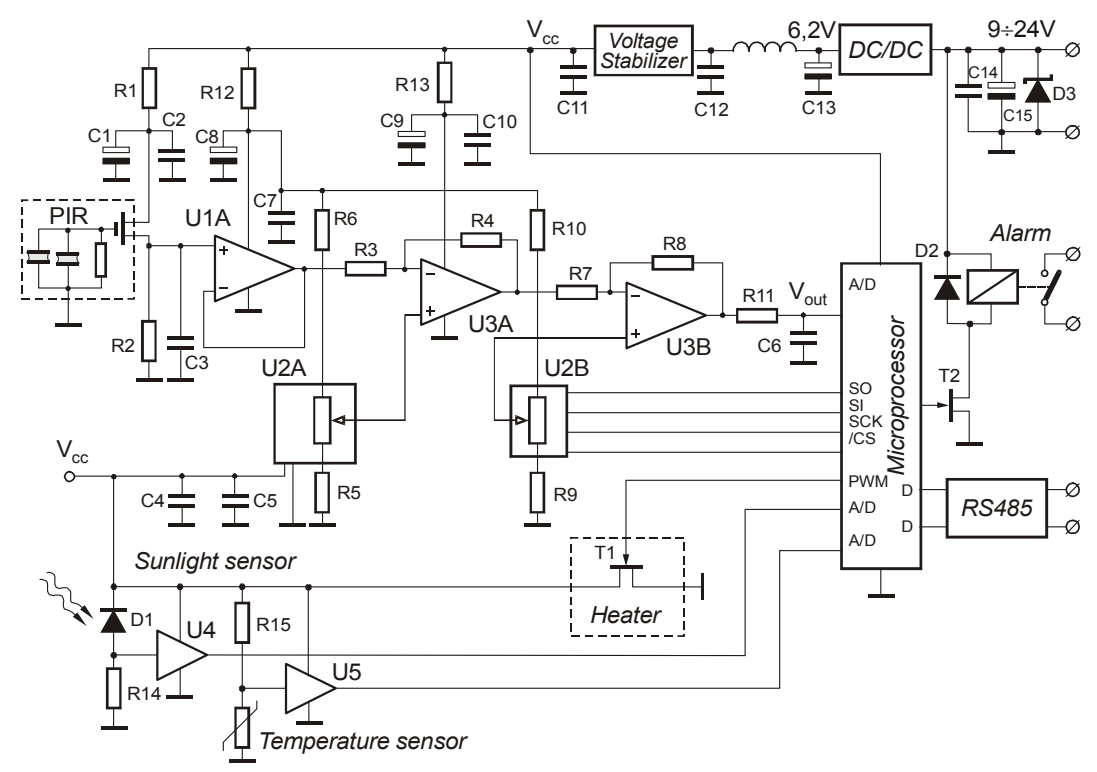

Figure 3: Simplified diagram of electronic circuit of PIR detector (one channel).

A system of DC amplifier with regulation (by means of digital electronic potentiometers) of a level of a constant component consists of three operation amplifiers. The first U1A amplifier, because of significant output resistance of a pyroelectric sensor operates in a follower system and two consecutive ones in the systems reversing a signal phase. The first stage of amplification is realized by the operation amplifier U3A. It is characterized with the signal amplification, $\mathrm{K} 1=-\mathrm{R} 4 / \mathrm{R} 3$. At its , + " input, a reference voltage is applied obtained from the resistance divider R5, R6, U2A (digital potentiometer) included between the supply voltage and the system ground.

Reference voltage (regulated by means of a digital electronic potentiometer) is used for elimination of a constant component of a signal obtained from a pyroelectric sensor. This component is significantly changed together with temperature changes occurring near a pyroelectric sensor (because of a change of the output current change of a field transistor dependent on temperature). Next, the signal is delivered to the second amplifying stage of the U3B operation amplifier the amplification of which is $\mathrm{K}=-\mathrm{R} 8 / \mathrm{R} 7$. At its "+" input, it has the reference voltage obtained from the R9, R10, U2B resistance divider (digital potentiometer). This voltage (regulated by means of a digital electronic potentiometer) protects the amplifier against saturation state. The output signal is filtered in the filter made from R11 and C6 elements. The electronic potentiometers, used in a preamplifier, are connected through a SPI series bus with a controlling processor which on the basis of the output signal values makes current adequate regulations (change in the value of potentiometer resistances) of the values of reference voltages of particular amplifying stages. 
Knowing the $N_{A}$ and $N_{B}$ settings (number of a selected level) of particular digital potentiometers, their total resistances $R_{A}$ and $R_{B}$ and the values of amplifications of particular $K 1, K 2$ preamplifier stages, the signal value $V_{O U T}$ at the amplifier output can be calculated:

$$
V_{\text {OUT }}=V_{I N} K 1 K 2-U_{p A} K 1 K 2-U_{p B} K 2
$$

where $V_{\mathrm{IN}}$ is the input voltage of the first amplifying stage, $U_{\mathrm{pA}}$ is the reference voltage of the first amplifying stage, and $U_{\mathrm{pB}}$ is the reference voltage of the second amplifying stage.

The values of reference voltages of particular amplifying stages for 8-bit resolution of digital potentiometers settings can be calculated from the relationship:

$$
\begin{aligned}
U_{p A} & =\frac{V_{c c}}{R 6+R_{A}+R 5}\left(R 5+\frac{N_{A}}{256} R_{A}\right) \\
U_{p B} & =\frac{V_{c c}}{R 10+R_{B}+R 9}\left(R 9+\frac{N_{B}}{256} R_{B}\right)
\end{aligned}
$$

where $V_{c c}$ is the supply voltage.

The $U_{p A}$ and $U_{p B}$ values are currently matched by a microprocessor ensuring adequate dynamic of the amplifier and preventing the saturation state of particular amplifying stages. If the output voltage of the amplifier will be measured and analyzed by a microprocessor, which in its operation algorithm considers the shift of levels (potentiometer settings), it can be shown that the voltage calculated (by a microprocessor) can be significantly higher than the supply voltage. It is an important virtue of this system.

Application of electronic potentiometers requires initial determination of their working ranges by means of external resistors. A better solution is application of digital-analogue converters instead of potentiometers.

In a sensor's electronic system, the system for measurement of sun radiation intensity was used. The information from this system is taken into account for in the intruder detection algorithm. The data from the temperature measurement system can be used for switching-on a miniature heater system mounted inside the PIR detector housing. A small increase in detector's interior temperature (in relation to ambient temperature) protects against water vapour deposition on optical elements (lens, concentrator mirrors, optical windows of sensors).

\section{Method of signal analysis}

An object in the sensor's "observation zone" (inspection zone) is detected when a conventional detection threshold is exceeded by a signal level at the detection system output, caused by IR emitted from an object. In order to minimize the probability of false alarms, an adaptation detection threshold should be determined "following up" the changing atmospheric conditions causing changes of "thermal scene" parameters [6]. 
762 Computational Methods and Experimental Measurements XIII
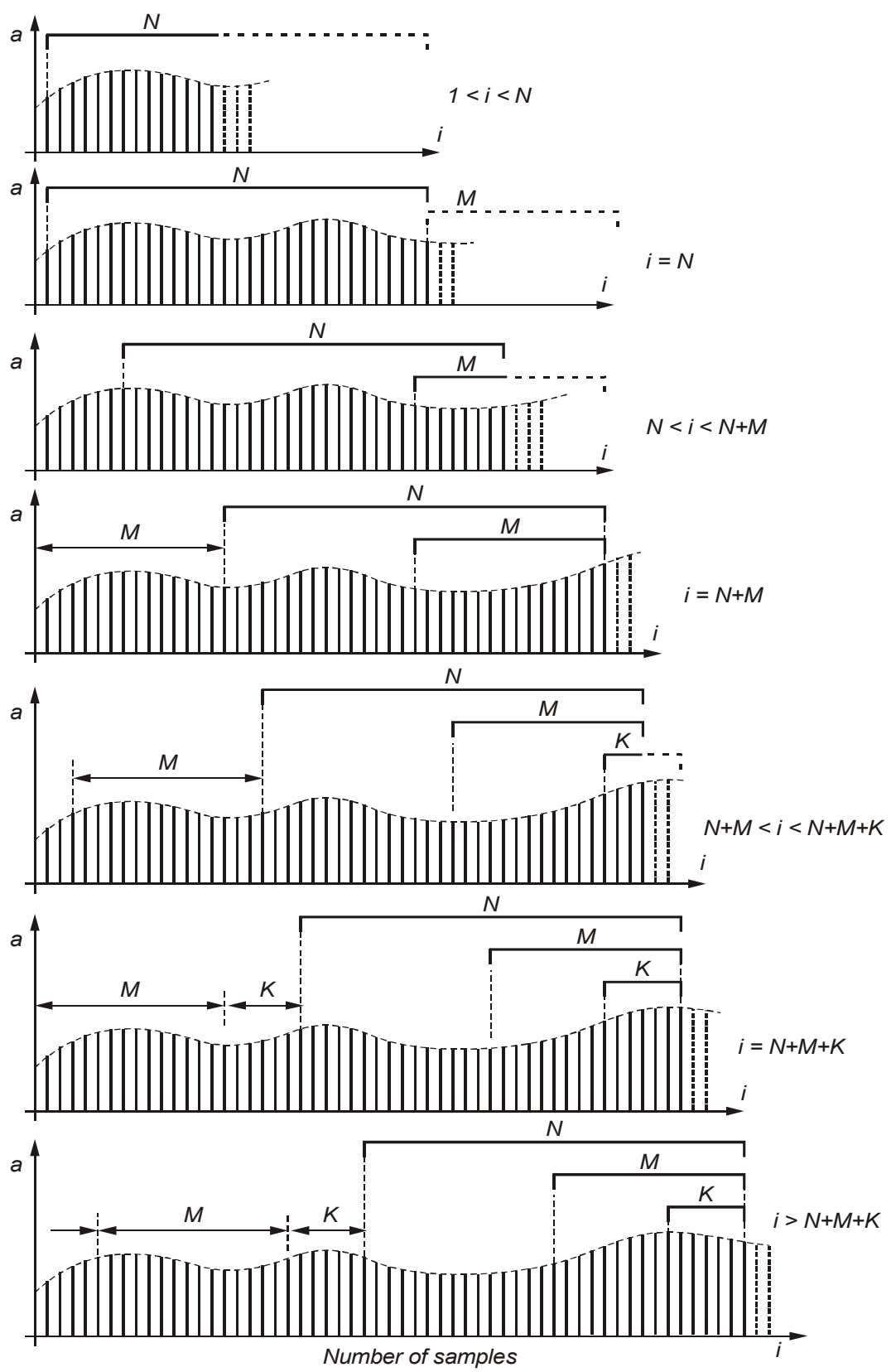

Figure 4: Method of creation of "time windows" in which samples of signals from pyroelectric sensors are analyzed. 
The analysis method is based on determination of an average moving in three "time windows" of a defined length [7]. The principle of formation of "time windows" is explained in Fig. 4. The windows are marked with $N, M$, and $K$ letters while $N>M>>K$ is assumed.

Signal processing is carried out in the following way. The signal from a detection set is sampled and the voltages of successive signal samples of instantaneous values $a_{i}$ are added. The time window is formed, containing $N$ samples, which in each consecutive cycle "shifts" by one sample. On the basis of an arithmetic average of voltage values of the samples in the window (Fig. 5), a value of the reference voltage level $\varphi_{i}$ is determined

$$
\varphi_{i}=\frac{1}{N}\left(a_{i-N+1}+a_{i-N}+\ldots+a_{i}\right) \text {, for } i>N
$$

where $i$ is the sample number which takes the values $i=1,2,3, \ldots$

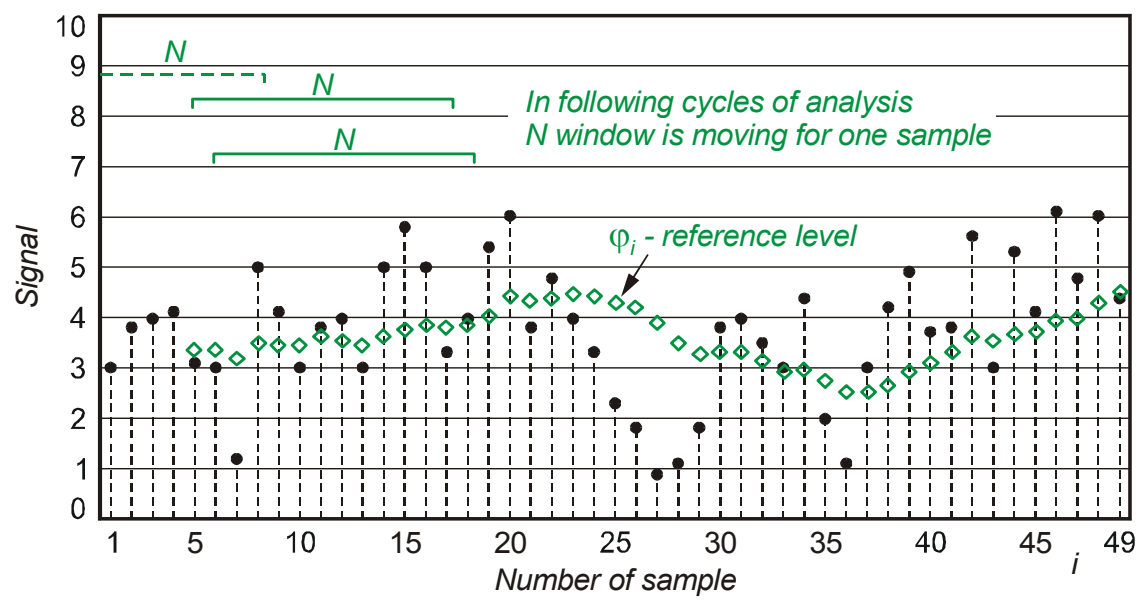

Figure 5: Sampled signal from a detection channel and the reference level $\varphi_{i}$ calculated according to Eq. (4).

In the next window $M$, that is in the previously formed window $N$ and containing $M$ samples (for $i \geq N+M$ ), the instantaneous values of the signal $\beta_{i}$ (Fig. 6) are determined as a difference of instantaneous values of voltages of consecutive samples of the signal and the reference level voltage $\varphi_{i}$

$$
\beta_{i}=a_{i}-\varphi_{i}, \text { for } i \geq N+M \text {. }
$$

For further calculations, the absolute value $\beta_{i}$ is taken as

$$
\left|\beta_{i}\right|=\left|a_{i}-\varphi_{i}\right|, \text { for } i \geq N+M \text {. }
$$




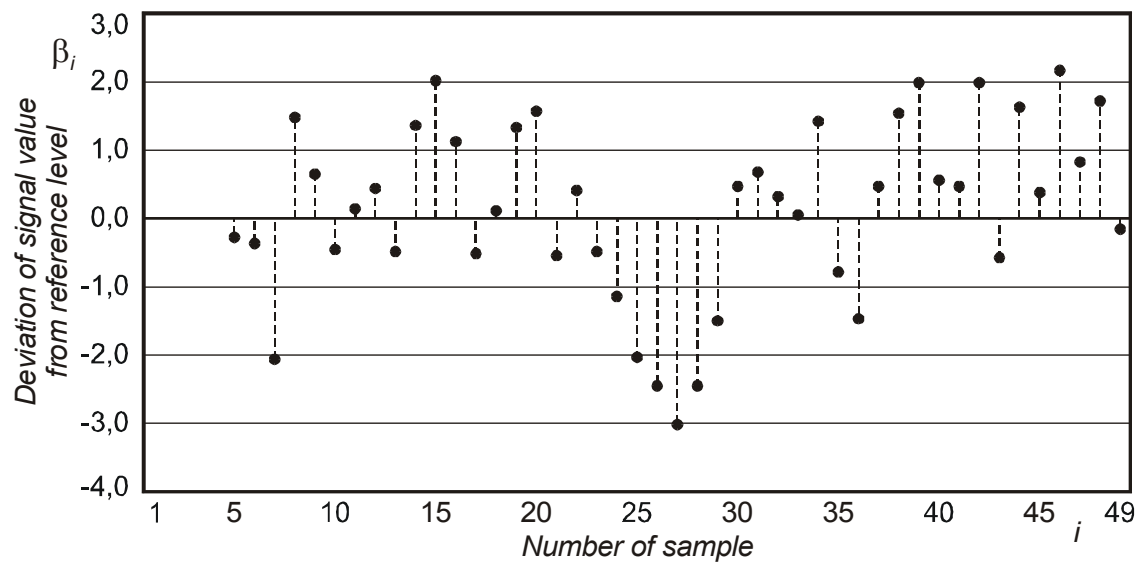

Figure 6: Instantaneous deviation of the signal $\beta_{i}$ from reference level calculated according to Eq. (5).

On the basis of such determined data, the instantaneous value of the detection threshold voltage $D_{i}$ which for $i>N+M$ is calculated as:

$$
D_{i}=A \frac{1}{M}\left(\left|\beta_{i-M+1}\right|+\left|\beta_{i-M}\right|+\ldots+\left|\beta_{i}\right|\right),
$$

where $A$ is the coefficient considering detector design parameters.

For typical solutions, the A coefficient takes the values $1 \div 5$. For PIR detectors of several detection zones and extended operation range, the A coefficient of the higher values for the zone being near detector is taken and A of the lower values is assumed for distant zones.

In order to diminish the influence of sun radiation, precipitation, and ambient temperature, the instantaneous corrected detection threshold $D_{k i}$ is determined:

$$
D_{k i}=D_{i} \cdot k_{s} \cdot k_{w} \cdot k_{t} \quad i>N+M
$$

where $k_{s}$ is the correction coefficient considering sun radiation, $k_{w}$ is the correction coefficient of precipitation influence, and $k_{t}$ is the correction coefficient of ambient temperature. The correction coefficient $k_{s}$ increases the level of a detection threshold in the case of sun illumination [8-10]. The values of changes in a detection threshold were determined experimentally from investigations on the influence of sun illumination level on the increase in both object temperature and background thermal noise. Because the object is also illuminated with sun radiation, which increases its equivalent temperature, increase in a detection threshold value does not cause a decrease in a sensor range and undesirable disturbances are eliminated. The value of the $k_{s}$ coefficient was taken as $1 \div 1.5$. 


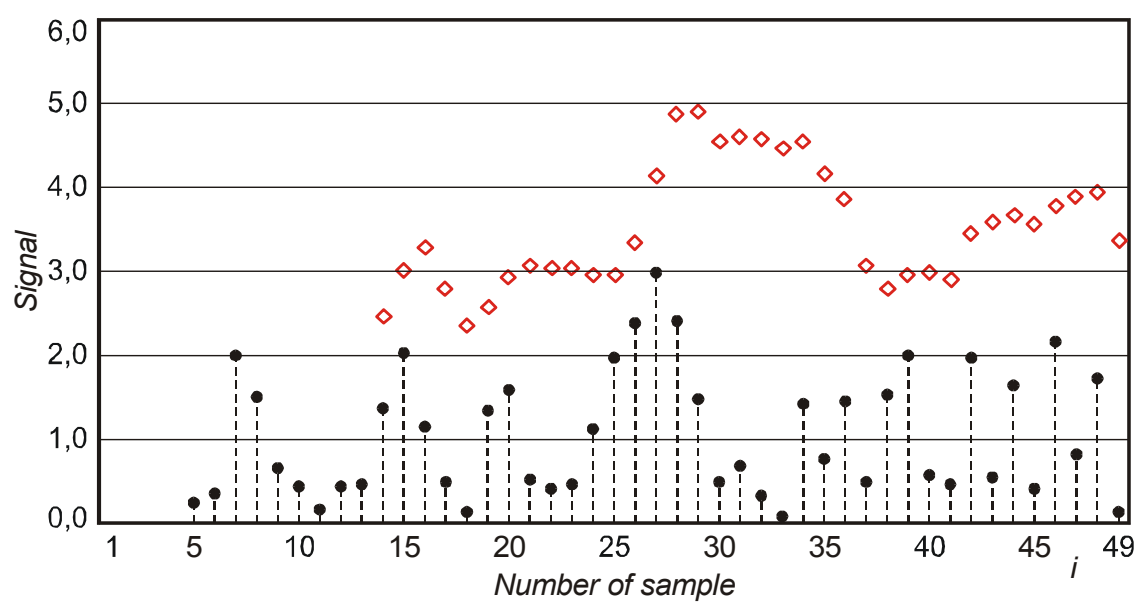

Figure 7: Instantaneous value of the detection threshold voltage $D_{i}$ and the absolute values of $\beta_{i}$ signal.

The $k_{w}$ coefficient causes a decrease in a detection threshold in the case of precipitation in the detection zone of the PIR detector. When intensity of precipitation (rain, snow) is higher, IR is much more attenuated which results in lower radiation reaching the detector, thus the signal at its output is weaker. When keeping the same value of a detection threshold, the sensor range is smaller. The $k_{w}$ coefficient is just to compensate the influence of precipitation in such a way as to keep the sensor range unchanged. The value of the $k_{w}$ coefficient was taken in the limits $0.7 \div 1$.

In the next step, the samples in the time window $K$ are analysed in which the parameter $\gamma_{i}$ is determined as an arithmetic average of the values of the signal samples $\left|\beta_{i}\right|$ :

$$
\gamma_{i}=\frac{1}{K}\left(\left|\beta_{i-K+1}\right|+\left|\beta_{i-K}\right|+\ldots+\left|\beta_{i}\right|\right), \text { for } i \geq N+M+K .
$$

An object is detected (Fig. 8) in the analysed signal when the parameter $\gamma_{i}$ is higher than the instantaneous value of the corrected detection threshold $D_{k i}$, i.e., when

$$
\gamma_{i}-D_{k i}>0
$$

In Fig. 8, also short lasting disturbances of high amplitudes are illustrated (samples 18 and 45) which are not interpreted as object detection. 


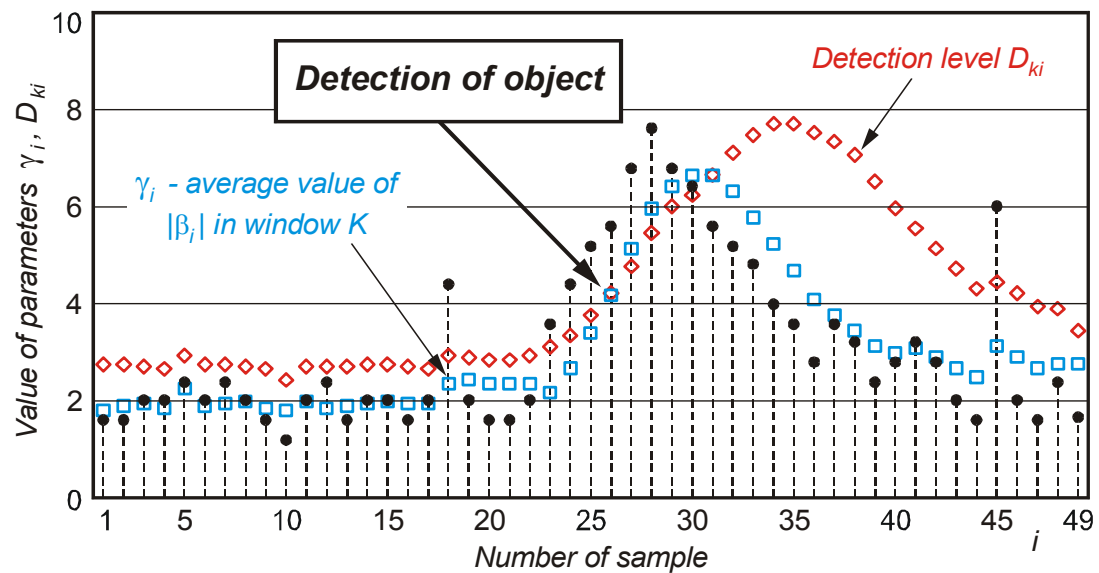

Figure 8: $\quad$ Object is detected when a value of $\gamma_{i}$ parameter exceeds a value of corrected detection threshold (in figure for samples No $26 \div$ No 31 ).

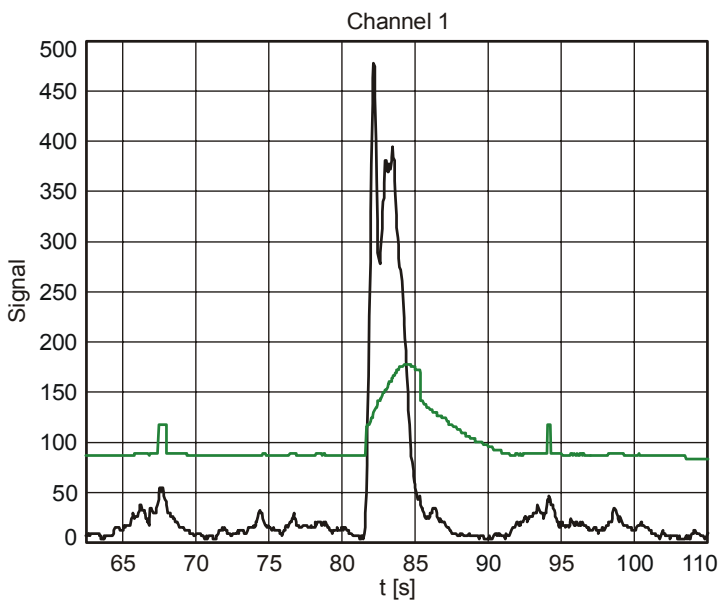

Figure 9: $\quad$ Signal in channel 1 of PIR detector recorded during night (ambient temperature $25^{\circ} \mathrm{C}$, velocity of human target $1 \mathrm{~m} / \mathrm{s}$, distance 140 meters).

\section{Results of range investigations}

The PIR detector is equipped with a data transmission path in the standard RS 485. For measurement results registration, special software developed for detector diagnostics was used. The software allows for signal registration from particular detection zones. In Figs. 9-11, the signals from the PIR detector are shown which were caused by a moving person in an inspection zone. 


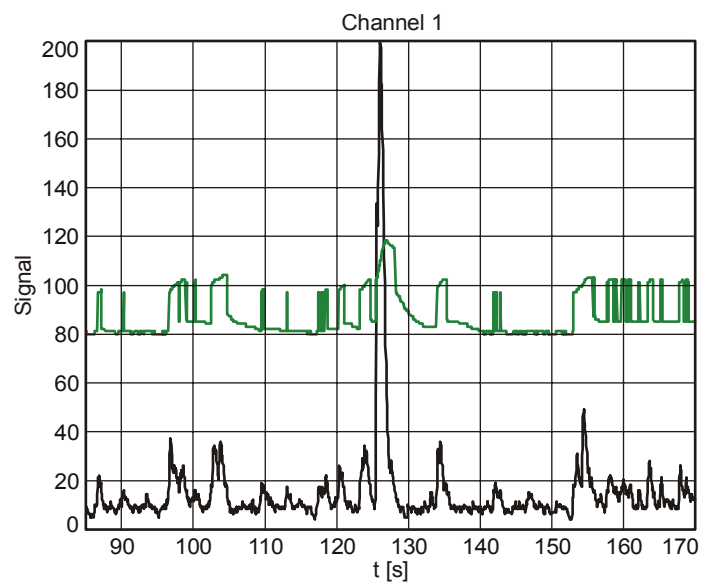

Figure 10: Signal in channel 1 of PIR detector recorded during sunny day (ambient temperature $28^{\circ} \mathrm{C}$, velocity of human target $5 \mathrm{~m} / \mathrm{s}$, distance 140 meters).

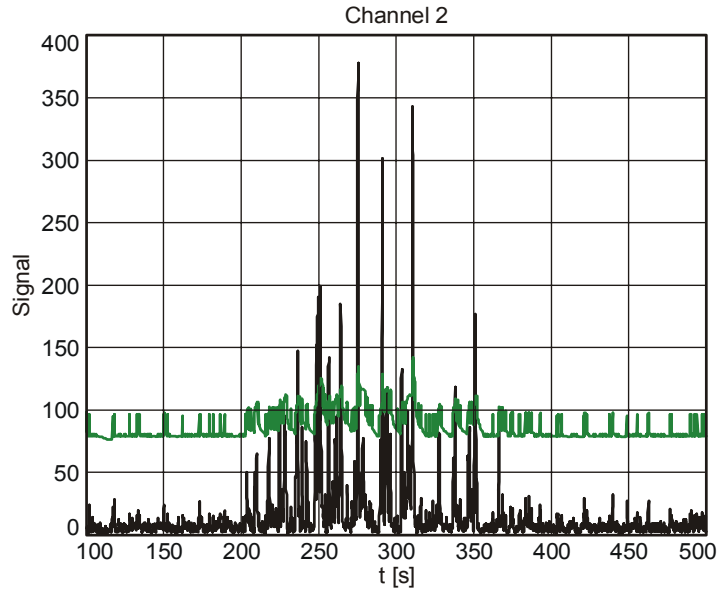

Figure 11: Signal in channel 2 of the PIR detector (ambient temperature $26^{\circ} \mathrm{C}$, velocity of crawling human target $0.1 \mathrm{~m} / \mathrm{s}$, distance 140 meters).

The investigation results, shown in Figs. 9-11, confirmed the proper operation of the PIR detector, in particular correctness and high efficiency of the signal processing method. Figure 10 shows that the detection threshold follows up (upper course) the thermal changes of a background which for this measurement case were caused by sun radiation. 


\section{Acknowledgement}

This work was financially supported by the Polish Ministry of Science and Higher Education in the frame of research project No 0005/T00/2005/28.

\section{References}

[1] ELTEC Instruments Inc.: Catalogue of firm products, P.O. Box 9610, Central Business Park, Daytona Beach, Florida 32120-9610, USA, 1996.

[2] Madura H., Sikorski Z., Polakowski H., Kastek M. Long-range passive IR sensor, Quantitative Infrared Thermography 5 QIRT'2000, Reims, France, 18-21.07.2000.

[3] Kastek M.: Method of objects detection in IR system of elongated detection zone. Doctors Thesis, Warsaw, The Military University of Technology Library, 2002 (in Polish).

[4] Madura H., Sikorski Z., Polakowski H., Kastek M. Automated stand for measurement of parameters of long-range passive IR sensor. Quantitative Infrared Thermography, Reims France, pp. 118-121, 2000.

[5] Kastek M., Madura H., Morawski M., Piatkowski T., Powiada E., Polakowski H. Test bed for measurement of angular parameters of passive infrared sensors. Infrared Physics \& Technology, Volume 49, No 3, pp. 198-201, 2007.

[6] Leach G. A single performance measure for perimeter intruder detection systems, European Conference on Security and Detection, Conference Publication No 437, pp. 114-119. 28-30.04. 1997.

[7] Madura H., Kastek M., Powiada E. Method of objects detection by means of IR sensors, Polish Patent Office, Patent Application P-360064, Warsaw 2003 (in Polish).

[8] Polakowski H., Piątkowski T., Madura H., Kastek M., Stojak Z., Powiada E., Chmielewski K., Kulewski R. Method of disturbances correction in IR receivers, Polish Patent Office, Patent Application P-352889, Warsaw 2002, (in Polish).

[9] Madura H., Kołodziejczyk M. Influence of sun radiation on results of noncontact temperature measurements in far infrared range. Opto-Electronics Review, No 13 (3), pp. 253-257, 2005.

[10] Madura H., Piątkowski T., Powiada E. Multispectral precise pyrometer for measurement of seawater surface temperature. Infrared Physics \& Technology Vol. 46 pp. 69-73, 2004. 